\title{
Effects of the $D_{1}$ Dopamine Receptor Agonist Dihydrexidine (DAR-0 I00A) on Working Memory in Schizotypal Personality Disorder
}

\author{
Daniel R Rosell ${ }^{1,2}$, Lauren C Zaluda ${ }^{1,3}$, Margaret M McClure ${ }^{1,3}$, M Mercedes Perez-Rodriguez $^{1,3}$, \\ K Sloan Strike ${ }^{1,3}$, Deanna M Barch ${ }^{4}$, Philip D Harvey ${ }^{5,6}$, Ragy R Girgis ${ }^{7,8}$, Erin A Hazlett ${ }^{1,3}$, \\ Richard B Mailman', Anissa Abi-Dargham ${ }^{7,8}$, Jeffrey A Lieberman ${ }^{7,8}$ and Larry J Siever*, , , \\ 'Department of Psychiatry, Icahn School of Medicine at Mount Sinai, New York, NY, USA; '2Department of Outpatient Psychiatry, \\ James J. Peters VA Medical Center, Bronx, NY, USA; ${ }^{3}$ Mental Illness Research, Education, and Clinical Center (MIRECC VISN 3), James J. Peters \\ VA Medical Center, Bronx, NY, USA; ${ }^{4}$ Department of Psychology, Washington University in St Louis, St Louis, MO, USA; ${ }^{5}$ Department of \\ Psychiatry, University of Miami Miller School of Medicine, Miami, FL, USA; ${ }^{6}$ Research Service, Bruce W. Carter VA Medical Center, Miami, FL, USA; \\ ${ }^{7}$ New York State Psychiatric Institute, New York, NY, USA; ${ }^{8}$ Department of Psychiatry, Columbia University Medical Center, New York, NY, USA; \\ ${ }^{9}$ Department of Pharmacology, Penn State University College of Medicine, Hershey, PA, USA
}

\begin{abstract}
Pharmacological enhancement of prefrontal $D_{1}$ dopamine receptor function remains a promising therapeutic approach to ameliorate schizophrenia-spectrum working memory deficits, but has yet to be rigorously evaluated clinically. This proof-of-principle study sought to determine whether the active enantiomer of the selective and full D, receptor agonist dihydrexidine (DAR-0I00A) could attenuate working memory impairments in unmedicated patients with schizotypal personality disorder (SPD). We performed a randomized, doubleblind, placebo-controlled trial of DAR-0 I00A ( $15 \mathrm{mg} / 150 \mathrm{ml}$ of normal saline administered intravenously over $30 \mathrm{~min}$ ) in medication-free patients with SPD $(n=16)$ who met the criteria for cognitive impairment (ie, scoring below the 25th percentile on tests of working memory). We employed two measures of verbal working memory that are salient to schizophrenia-spectrum cognitive deficits, and that clinical data implicate as being associated with prefrontal $D_{\text {I }}$ availability: (I) the Paced Auditory Serial Addition Test (PASAT); and (2) the $\mathrm{N}$-back test (ratio of 2-back:0-back scores). Study procedures occurred over four consecutive days, with working memory testing on Days I and 4, and DAR-0I00A/placebo administration on Days 2-4. Treatment with DAR-0I00A was associated with significantly improved PASAT performance relative to placebo, with a very large effect size (Cohen's $d=1.14$ ). Performance on the $\mathrm{N}$-back ratio was also significantly improved; however, this effect rested on both a non-significant enhancement and diminution of 2-back and 0-back performance, respectively; therefore interpretation of this finding is more complicated. DAR-0I00A was generally well tolerated, with no serious medical or psychiatric adverse events; common side effects were mild to moderate and transient, consisting mainly of sedation, lightheadedness, tachycardia, and hypotension; however, we were able to minimize these effects, without altering the dose, with supportive measures, eg, co-administered normal saline. Although preliminary, these findings lend further clinical support to the potential of $D_{1}$ receptor agonists to treat schizophrenia-spectrum working memory impairments. These data suggest a need for further studies with larger group sizes, serum DAR-0I00A levels, and a more comprehensive neuropsychological battery.

Neuropsychopharmacology (2015) 40, 446-453; doi:I 0.1038/npp.2014.192; published online 27 August 20I4
\end{abstract}

\section{INTRODUCTION}

Cognitive deficits are a critical determinant of functional outcomes in schizophrenia and related psychiatric conditions (Green, 2006), yet they remain relatively resistant to standard treatment (Keefe, 2007) approaches. A number of experimental agents with preclinical promise have, unfortunately, yielded disappointing results in clinical trials

*Correspondence: Dr LJ Siever, James J. Peters Department of Veterans Affairs Medical Center, I 30 West Kingsbridge Road, Room 6A-44, Bronx, NY 10468, USA, Tel: +I 7185849000 ext. 5227, Fax: + 718364 3576, E-mail: larry.siever@va.gov

Received I 8 January 20 I4; revised 30 June 20 I4; accepted I July 20 I4; accepted article preview online 30 July 2014
(Buchanan et al, 2011; Egan et al, 2013; Harvey, 2009; Velligan et al, 2012). Numerous studies have established that compromised working memory function is a central component of schizophrenia-spectrum cognitive deficits (Goldman-Rakic, 1994). Therefore, targeting the mediators of working memory, particularly those with pathophysiological significance, is an essential approach to develop effective therapeutics for the cognitive impairments of the schizophrenia spectrum.

There is an abundant experimental literature demonstrating the critical role of the $D_{1}$ dopamine receptor in the modulation of working memory in the dorsolateral prefrontal cortex (DLPFC) (Goldman-Rakic et al, 2004). Furthermore, frontocortical $D_{1}$ receptor availability has 
been shown to be relatively increased in antipsychotic-naive patients with schizophrenia; and this has been proposed to reflect compensatory, albeit insufficient, $\mathrm{D}_{1}$ upregulation in response to possible cortical hypodopaminergia in the schizophrenia spectrum (Abi-Dargham, 2003). Therefore, pharmacological enhancement of $D_{1}$ receptor activity is a highly promising therapeutic mechanism for the amelioration of schizophrenia-spectrum cognitive deficits. Importantly, however, there have been barriers to the development of pharmacological agents with $\mathrm{D}_{1}$ agonist activity (Mailman et al, 2001; Zhang et al, 2009), and therefore enhancement of working memory in the schizophrenia spectrum via direct $\mathrm{D}_{1}$ stimulation has yet to be studied definitively in the clinical setting.

Although it was recognized by the late 1970s that there were two major populations of dopamine receptors (Garau et al, 1978; Kebabian and Calne, 1979), the prototypical $\mathrm{D}_{1}$ agonist SKF38393 (Setler et al, 1978) was of limited experimental utility largely due to the fact that it had low intrinsic activity (Gilmore et al, 1995; Watts et al, 1993, 1995). It was a decade before dihydrexidine (DHX), the first selective and full $\mathrm{D}_{1}$ agonist, was described (Lovenberg et al, 1989). DHX exhibits more than ten-fold higher affinity and potency at the $D_{1}$ receptor than dopamine, and full intrinsic activity in every system in which it has been tested to date (Brewster et al, 1990; Mottola et al, 1992; Salmi et al, 2004; Watts et al, 1995). DHX has about ten-fold selectivity for the $\mathrm{D}_{1}$ vs the $\mathrm{D}_{2}$ receptor (Mottola et al, 1992), and at the $\mathrm{D}_{2}$ receptor it is functionally selective, with high bias for regulation of adenylate cyclase vs potassium channels (Gay et al, 2004; Kilts et al, 2002; Mailman, 2007; Mottola et al, 2002). When DHX entered development in 2002, it was assigned the label DAR-0100, and the active (both at $\mathrm{D}_{1}$ and $\mathrm{D}_{2}$ ) diastereomer (Knoerzer et al, 1994; Mottola et al, 1996) was assigned the label DAR-0100A.

Although discovered more than 20 years ago, DHX remains the prototypical full, selective $D_{1}$ agonist. DHX has been shown to enhance working memory in animal models in normal and pathological states (Arnsten et al, 1994; Schneider et al, 1994), yet clinical testing of DHX has been limited for at least two reasons: First, the drug has a very short duration of action, and can only be given parenterally in currently available formulations. Second, an initial Phase I/IIa study in Parkinson's disease subjects showed some efficacy, but also resulted in profound hypotension (Blanchet et al, 1998). Later developmental work, however, demonstrated that the drug could be used safely (George et al, 2007), and this provided the foundation for an NIMHsponsored Cooperative Drug Development Group project (awarded to our Columbia University collaborators) that synthesized the active enantiomer, DAR-0100A, and obtained an Investigational New Drug application to optimize clinical infusion parameters, and examine the effects of DAR-0100A on cognitive function in schizophrenia and related conditions. Our group subsequently obtained an Investigational New Drug application to examine DAR-0100A in the schizophrenia spectrum, as well.

Evaluating the efficacy of novel agents (eg, DAR-0100A) to attenuate cognitive impairment in schizophrenia is made difficult by factors such as concurrent/past medication, stage of illness and prior psychotic episodes, relatively global cognitive deficits, and chronic psychosocial stress related to profound functional impairment. One approach to addressing these confounds is to study patients with schizotypal personality disorder (SPD), a schizophreniaspectrum condition that manifests qualitatively similar working-memory deficits that are narrower in scope and severity (Mitropoulou et al, 2002, 2005). Our prior studies of patients with SPD have shown dopaminergically mediated improvement of schizophrenia-spectrum cognitive deficits. This effect was first demonstrated with amphetamine (Siegel et al, 1996; Kirrane et al, 2000) which increases catecholaminergic activity globally, and more recently with pergolide (McClure et al, 2010), a mixed $\mathrm{D}_{2} / \mathrm{D}_{1}$ agonist with partial $\mathrm{D}_{1}$ agonist activity. No published clinical studies to date have demonstrated the efficacy of selective $\mathrm{D}_{1}$ agonists to mitigate schizophrenia-spectrum cognitive impairments.

Therefore, we have examined whether treatment with DAR-0100A could ameliorate the schizophrenia-spectrum cognitive deficits of unmedicated patients with SPD using two tests of verbal working memory: the Paced Auditory Serial Addition Test (PASAT) and $N$-back tests. We specifically selected these two tests of working memory because they consistently reveal cognitive impairments of patients with SPD (Mitropoulou et al, 2002; McClure et al, 2008), and because of the relationship between greater frontocortical $\mathrm{D}_{1}$ availability (which may reflect $\mathrm{D}_{1}$ understimulation) and degree of impairment of PASAT (Thompson et al, 2014) and $N$-back performance (AbiDargham et al, 2002) in the schizophrenia spectrum. Furthermore, among a broad array of cognitive function tests, we have shown that PASAT performance accounts for a substantial component of the variance between SPD patients and healthy controls, implicating the salience of this test for assessing cognitive impairment in SPD (Mitropoulou et al, 2005).

\section{MATERIALS AND METHODS}

\section{Participants}

The study was approved by the Institutional Review Boards of the Mount Sinai Medical Center and the James J. Peters Bronx Veterans Affairs Medical Center. Participants provided written informed consent after explanation of study procedures. Participants were recruited from the local population through newspaper advertisements and the internet. All participants underwent a medical clearance, consisting of a medical history by a study physician, physical exam, basic blood and urine tests, and electrocardiogram. All participants were free of significant medical/neurological problems, and were not pregnant or nursing. Sixteen participants (11 male (M) 5 female $(\mathrm{F})$; mean age $=35.9$ years $(S D=12.2$, range $=20-55)$ met the criteria for SPD. The Structured Clinical Interview for DSM-IV Axis I Disorder was used for Axis I diagnoses, and the Structured Interview for DSM-IV Personality Disorders was used for Axis II diagnoses.

Participants with active suicidal ideation, as well as participants who met the criteria for major psychotic disorders (such as bipolar I or schizophrenia), or participants who currently met the DSM-IV-TR criteria for a major depressive episode were excluded from participation and referred for appropriate treatment. Participants had neither abused 
illicit substances nor alcohol within the past 6 months, nor had a past history of substance dependence. All patients were negative on a urine toxicology screen to rule out current use of common substances of abuse. Participants were excluded if they did not exhibit cognitive impairment, as defined by scoring at or below the 25th percentile of published scores for healthy participants on at least one test of working-memory battery that included the PASAT, $\mathrm{N}$-back test, and Dot test. Of note, the Dot test-a measure of visuospatial working-memory-was originally included as a secondary measure; however, too few patients completed the Dot test, and therefore it was ultimately not included in our final analyses.

\section{Treatment Design}

The protocol occurred over four consecutive days that included baseline cognitive testing (see Cognitive Assessments, below), and urine drug (and pregnancy, for women of child-bearing age) screening on Day 1 . On days 2-4, an intravenous catheter was placed by a qualified nursing staff member of the Clinical Research Unit of the Mount Sinai Medical Center, and either DAR-0100A ( $15 \mathrm{mg} / 150 \mathrm{ml}$ of normal saline (NS)) or placebo ( $150 \mathrm{ml}$ of NS) was intravenously infused at a rate of $300 \mathrm{ml} / \mathrm{h}$. Additionally, $133 \mathrm{ml}$ of intravenous. NS was co-infused (beginning $5 \mathrm{~min}$ prior to and ending $5 \mathrm{~min}$ after DAR-0100A/placebo infusion) at a rate of $200 \mathrm{ml} / \mathrm{h}$. Repeat cognitive testing (beginning $\sim 20 \mathrm{~min}$ after the start of DAR-0100A/placebo infusion) was performed on days 2 and 4 . Serial liver function testing was also performed because transient, clinically insignificant transaminase increases had been seen in three subjects in early studies at Columbia.

Participants were randomized in a double-blind manner to receive either DAR-0100A or placebo throughout the three days of infusion. Day 1 procedures (other than baseline liver function testing) took place in the research office of the Mood and Personality Disorders Group. Procedures for Days 2-4 occurred in the Clinical Research Unit of the Mount Sinai Medical Center. On days 2-4, participants were semi-recumbent in a standard hospital bed with the upper body raised to $\sim 45^{\circ}$ during cognitive testing. Participants were monitored by a study physician and the Clinical Research Unit nursing staff; vital signs were obtained every 5 min during DAR-0100A/placebo infusion, and every $15 \mathrm{~min}$ for $1 \mathrm{~h}$ after completion of the infusion.

\section{Cognitive Assessments}

Rationale for choice of cognitive assessments. Based on our prior studies in SPD and schizophrenia, we selected two tests of verbal working memory that we have demonstrated are impaired in SPD (McClure et al, 2008; Mitropoulou et al, 2002, 2005), and that evidence suggests are related to frontocortical $\mathrm{D}_{1}$ availability (Abi-Dargham et al, 2002; Thompson et al, 2014): (1) the PASAT ; and (2) the N-back test. (As indicated above, the Dot test-a measure of visuospatial working memory-was originally included; however, data were available for too few participants.) Of note, we did not include neuropsychological tests that formally assess cognitive processes other than working memory (eg, attention, executive function, declarative memory, processing speed) given the short half-life of DAR-0100A, and the anticipated narrow window of opportunity for identifying a therapeutic effect.

PASAT. The PASAT is a test of auditory/verbal working memory. Participants are presented with a series of numbers (50 numbers at the rate of one digit per $2 \mathrm{~s}$ ) by audiotape, and are asked to add each adjacent pair of numbers and report the sum. The total number of correct responses (range 0-49) was computed. We then calculated ratio scores (post-treatment minus baseline/baseline) in order to control for baseline differences in performance. This ratio score was the dependent variable in this study.

$\mathrm{N}$-back test. The N-back test is an assessment of visual/ verbal working memory. We employed two conditions: (a) the 0-back-a control condition that primarily assesses sustained attention-in which participants are instructed to press a target button when presented with the letter ' $\mathrm{X}$ '; and (b) the 2-back - which requires working memory as well as sustained attention-in which participants are instructed to press a target button when the letter appearing is the same as the letter that appeared two previous screens back. In order to account for individual differences in attentional functioning, $\mathrm{N}$-back test scores are represented in terms of a 2-back: 0-back ratio. We again calculated ratio scores (posttreatment minus baseline/baseline), in order to control for baseline differences in performance. This ratio score was the dependent variable.

\section{Data Analysis}

Between-group $t$-tests were used to examine potential group (DAR-0100A and placebo) differences of baseline scores and percent change scores (post-treatment minus pre-treatment). A two-group chi-square test was used to examine potential group differences of the baseline gender ratios.

\section{RESULTS}

The average age of the 16 participants ( $11 \mathrm{M}, 5 \mathrm{~F}$ ) who were diagnosed with SPD and completed the study was 35.9 years $(\mathrm{SD}=12.2$, range $=20-55) \quad($ Table 1$)$. The DAR-0100A $(n=8)$ and placebo $(n=8)$ groups were comparable with respect to age and gender (Table 1). The two groups did not differ significantly on baseline cognitive scores (Table 2).

Comparisons of change scores of our tests of verbal working memory between the two groups are illustrated in Table 2. (Note that one of the participant's $N$-back data in the DAR-0100A group was lost owing to computer error,

Table I Demographics of Subjects

\begin{tabular}{lccc}
\hline & Placebo group & DAR-0 I00A group & $\boldsymbol{p ~ v a l u e ~}^{\mathbf{a}}$ \\
\hline Age (years) & $32.1(10.4)$ & $39.8(13.3)$ & 0.22 \\
$n$ (male:female) & $5: 3$ & $6: 2$ & 1.0 \\
\hline
\end{tabular}

Note. Values represent mean (SD).

${ }^{a} p$ values derived from $t$-tests, except for M:F ratio comparisons, chi-square was used. 
Table 2 Comparison of Change Scores Between DAR-0I00A and Placebo Groups

\begin{tabular}{|c|c|c|c|c|c|c|c|c|c|}
\hline \multirow[t]{2}{*}{ Cognitive test } & \multicolumn{2}{|c|}{ Placebo } & \multicolumn{2}{|c|}{ DAR-0I00A } & \multicolumn{2}{|c|}{ Change score (\%) } & \multicolumn{3}{|c|}{ Group comparison } \\
\hline & Baseline & Post-infusion & Baseline & Post-infusion & Placebo & DAR-0I00A & Cohen's d & $t(d f)$ & $p$ \\
\hline \multicolumn{10}{|l|}{$\mathrm{N}$-back ${ }^{\mathrm{b}, \mathrm{c}}$} \\
\hline O-back & $0.95(0.07)$ & $0.94(0.06)$ & $0.94(0.06)$ & $0.89(0.09)$ & $-1.03(8.83)$ & $-5.54(6.74)$ & -0.57 & $1.10(13)$ & 0.29 \\
\hline
\end{tabular}

Abbreviation: PASAT, paced auditory serial addition test.

Note. Values represent mean (SD).

aPASAT values represent number of correct answers-highest possible score is 49.

${ }^{b} \mathrm{~N}$-back values represent proportion of correct answers.

$c_{n}=7$ for DAR-0 I00A N-back owing to loss of one participant's data because of computer error.



Figure I Percent-change scores for the PASAT, 0-back, 2-back, and $\mathrm{N}$-back ratio (2-back/0-back). Scores for the PASAT and N-back ratio were significantly greater in the group treated with DAR-0I00A compared to placebo. Error bars represent the standard error of the mean.

and therefore could not be included in the $\mathrm{N}$-back analysis.) Compared with placebo (Figure 1), the DAR-0100A group exhibited significantly greater improvement in the PASAT $(t=-2.27, \mathrm{df}=14, p=0.04)$, with a very large effect size (Cohen's $d=1.14$ ).

The effect of DAR-0100A treatment on $N$-back performance was less clear than that of the PASAT, however. As shown in Table 2, the $\mathrm{N}$-back ratio was significantly improved in the DAR-0100A group relative to placebo $(t=-2.23$, $\mathrm{df}=13, p=0.04)$. Similar to the effect on the PASAT, improvement of the $\mathrm{N}$-back ratio was associated with a very large effect size (Cohen's $d=1.14$ ). However, this effect on the $\mathrm{N}$-back ratio was determined by both a non-significant improvement, with a moderate effect size (Cohen's $d=0.46$ ), in 2-back performance, as well as a non-significant decrease in 0-back performance, also with a moderate effect size (Cohen's $d=-0.57$ ). Given the contribution of the nonsignificant decrease in 0-back performance to the improvement of the $\mathrm{N}$-back ratio, the significance of improved $\mathrm{N}$-back ratio performance is less clear than that of the PASAT. We suspect that side effects may have non-specifically led to a possible diminution of non-delayed cognitive processes that underlie 0-back performance. Improvement of $\mathrm{N}$-back ratio performance may suggest DAR-0100A enhanced working memory despite some attenuation of non-delayed perceptual/attentional processes.

DAR-0100A was relatively well tolerated, with no clinically significant adverse events. Side effects were mild to moderate and transient, consisting of lightheadedness, sedation, subjective facial flushing, subjective racing heart, moderate tachycardia, and clinically insignificant decreases in systolic blood pressure. No immediate or delayed psychiatric side effects were associated with DAR-0100A treatment, and no liver function testing changes occurred in any of the participants.

\section{DISCUSSION}

This is the first published study to examine the effects of a selective $\mathrm{D}_{1}$ agonist on salient measures of working memory function in a clinical population with schizophrenia-spectrum cognitive deficits without important confounds such as concurrent psychotropic medication, psychosis, substance abuse, and pervasive functional impairment. While our findings must be interpreted with caution, they do suggest that pharmacological enhancement of $D_{1}$ receptor activity may mitigate schizophrenia-spectrum working memory impairments. Important limitations of our study that must be taken into consideration are small sample size, the lack of serum drug concentrations, and the fact that improvement on the $\mathrm{N}$-back ratio in the DAR-0100A-treated group was due to a non-significant improvement in the 2-back and a non-significant decline of 0-back performance.

Treatment with DAR-0100A, under our optimized infusion parameters, was not associated with any dose-limiting side effects or significant medical sequelae. DAR-0100A was generally well tolerated, and side effects were transient, consisting primarily of facial flushing, lightheadedness, sedation, subjective racing heart, as well as minor increases in heart rate, and clinically insignificant hypotension, all of which resolved prior to or soon after completion of drug infusion. No serious cardiovascular or hepatic-function abnormalities occurred, and there were no acute or delayedonset psychiatric side effects. Specifically, there was no evidence of depression, mania, psychosis, agitation, anxiety, or confusion/disorientation on the days of drug infusion or at a 1-2-week follow-up. 
The first reported studies of DHX effects on cognitive function were performed in primates, and demonstrated cognitive enhancement in young, aged, and reserpinetreated animals (Arnsten et al, 1994), as well as chronic, low-dose MPTP-treated primates (Schneider et al, 1994). Furthermore, in rodent models, DHX also was shown to improve both native processes and reverse scopolamineinduced deficits (Steele et al, 1997), as well as attenuate PCP-induced deficits of NMDA-R activity (Lei et al, 2009).

As noted earlier, there have been two prior reported clinical studies with DHX: The first, in Parkinson's disease (Blanchet et al, 1998), showed anti-parkinsonian efficacy in one of four patients who received the highest dose $(35 \mathrm{mg} /$ $15 \mathrm{~min}$, intravenously.), but clinically significant hypotension occurred that was dose limiting despite lengthening the infusion time from 15 to $120 \mathrm{~min}$. Almost a decade later, administration of a single, subcutaneous dose of $20 \mathrm{mg}$ of DHX was found to be well tolerated in patients with schizophrenia who were medicated with risperidone (George et al, 2007). In this latter study, DHX treatment was associated with increases in prefrontal, as well as temporal and parietal cortical, perfusion ( $\mathrm{Mu}$ et al, 2007), and a significant improvement on a 2-back task, but only on the second day after treatment (Johnson, George, et al, personal communication). The latter result is difficult to resolve because of experimental confounds (eg, neuropsychological testing delayed for many hours after drug, etc.). The current study had several design advantages: First, we used a somewhat higher active dose (ie, $15 \mathrm{mg}$ of the active enantiomer DAR-0100A rather than $20 \mathrm{mg}$ of the racemate); cognitive testing was done soon after completion of DAR$0100 \mathrm{~A}$ infusion; neuropsychological tests were used that were particularly salient to schizophrenia-spectrum working-memory deficits; and patients were not receiving concurrent neuroleptics, nor did they suffer from pervasive functional impairment.

As with any drug, the question is whether the effect we have found with DAR-0100A can be ascribed solely to the activation of $D_{1}$ receptors. There are two primary off-target receptors that should be considered in the actions of DHX: the dopamine $\mathrm{D}_{2}$, and the $\alpha_{2}$-adrenergic receptor (Mottola et al, 2002). The one of greater importance is the $\mathrm{D}_{2}$ as DHX is only 10 -fold $\mathrm{D}_{1}: \mathrm{D}_{2}$ selective (Mottola et al, 2002). As noted earlier, however, DHX has unique functionally selective properties at $\mathrm{D}_{2}$ receptors, having full agonist effects at $\mathrm{D}_{2}$-dependent inhibition of adenylate cyclase $v s$ little or no intrinsic activity at $G$ protein-coupled inwardly rectifying potassium channels (Gay et al, 2004; Kilts et al, 2002; Mailman, 2007; Mottola et al, 2002). This probably explains why, even at very high doses, DHX does not have amphetamine-like actions in vivo (Darney et al, 1991) as would be expected from a drug activating both $\mathrm{D}_{1}$ and $\mathrm{D}_{2}$ receptors. Moreover, the turning induced by DHX in unilateral 6-OHDA rats is completely blocked by selective $\mathrm{D}_{1}$ antagonists, but not decreased by $\mathrm{D}_{2}$ antagonists (in contrast to the typical $\mathrm{D}_{2}: \mathrm{D}_{1}$ agonist apomorphine where each type of antagonist will attenuate the turning). In addition, DHX lessens psychomotor activity (an effect attenuated by $\mathrm{D}_{1}$, but not by $\mathrm{D}_{2}$, antagonists), actually antagonizing amphetamine-induced increases in motor activity, and causes immediate early-gene expression changes in the medial prefrontal cortex that are sensitive to $D_{1}$ but not $D_{2}$ antagonists (Isacson et al, 2004).
The other potentially important off-target locus is the $\alpha_{2}$ adrenergic receptor. It is not known what the functional characteristics of DHX are at this receptor, but we do know the drug is $\sim 50$-fold $D_{1}: \alpha_{2}$ selective. PET studies have shown that $\mathrm{D}_{2}$ occupancy is unlikely to be important with clinical doses of DHX (Slifstein et al, 2011), and because the $\alpha_{2}$-adrenergic affinity is five times lower than the $D_{2}$, it seems unlikely that the current data were affected by $\alpha_{2}$-adrenergic effects. Nonetheless, more detailed future studies with DHX and with newer $\mathrm{D}_{1}$ agonists will be needed to provide a conclusive test of this hypothesis.

It is also important to take into consideration that the animal studies of working-memory functional effects of $D_{1}$ agonists demonstrate an inverted-U-shaped dose-response curve rather than a plateauing curve. Based on the published pharmacology of DAR-0100A in humans, the doses we used result in very low blood concentrations that are almost certainly in the ascending phase of the doseresponse curve of this drug, being less than those that cause cognitive enhancement in non-human primates (Arnsten et al, 1994; Schneider et al, 1994). Nonetheless, this assumption will need to be tested in further studies in which a therapeutic range for DAR-0100A and other $D_{1}$ agonists is determined, as this has significant implications for the clinical utility of this class of drug.

Although there have been many selective full $\mathrm{D}_{1}$ agonists discovered during the past 25 years, only two compounds (DHX and ABT-431) have resulted in reports of clinical studies. ABT-431 is a prodrug of A-86929 that has in vitro pharmacology almost identical to DHX (Michaelides et al, 1995; Mottola et al, 1992). The data from both compounds allow us to speculate on other side effects that are attributed to dopamine agonists as a general class, including nausea, the exacerbation of psychosis, and drug-abuse-related behaviors. There is a sizable literature that suggests that nausea and psychosis exacerbation are largely mediated by $\mathrm{D}_{2}$ receptors. The issue of drug abuse liability is more complex as some studies in murine species suggest $D_{1}$ agonists have potential liability (Graham et al, 2007). On the other hand, it has been reported that ABT-431 actually decreased cocaine craving (Giardina and Williams, 2001), suggesting that clinically this will not be problematic.

A number of important study limitations need to be addressed: The sample size was modest; we did not assess serum drug concentrations; and cognitive domains other than those related to working memory (such as attention, executive function, and declarative memory) were not examined. Although we did collect serial blood samples during the 2nd day of drug/placebo infusion, these will not be analyzed until a later date once we have accrued enough samples to send out for analysis. In our current funded study, however, we are assessing a broad array of cognitive processes, and therefore we will be able to assess the specificity of effects of DAR-0100A.

An additional matter worthy of addressing is that improvement of the $\mathrm{N}$-back ratio was due to non-significant increases and decreases, respectively, of 2-back and 0-back performance. Taking into account that performance on the PASAT was significantly improved in the DAR-0100Atreated group, a plausible explanation for the mixed result on the $\mathrm{N}$-back is that working-memory function may have been enhanced, despite a possible attenuation of 
non-delayed attentional/perceptual processes. It is also possible that the $N$-back (a visual, verbal working-memory task) is not as sensitive to enhancement by DAR-0100A as is the PASAT (an auditory, verbal working-memory task). This is consistent with our recent finding that frontocortical $\mathrm{D}_{1}$ receptor availability in patients with SPD was negatively correlated with PASAT performance, but not that of the 2-back (Thompson et al, 2014). We speculate that any attenuation of non-delayed attentional/perceptual processes (eg, 0-back performance) in the DAR-0100A group may have been due to side effects such as sedation, and lightheadedness. However, it is also possible that the 15-mg dose was too high and eroded cognitive improvement, given the inverted- $U$ dose-response function of $D_{1}$ agonists. This issue could be addressed in future studies where side effects are minimized by supportive measures, or lower doses are assessed for effects on cognition.

Although side effects were mild, transient, and without significant clinical impact, their potential for breaking the blind is an additional study limitation. Anecdotal evidence from our Columbia colleagues (Girgis, personal communication) suggests participants were unable to correctly identify whether they were receiving DAR-0100A or placebo. Furthermore, in this study, the physician who performed clinical monitoring did not administer the workingmemory tests, and research personnel who did administer neuropsychological testing were not monitoring the side effects and vital signs. Nevertheless, we cannot rule out the possibility that failure to absolutely maintain the blind may have confounded our results.

In an effort to confirm our findings and address these limitations, we are currently conducting a large, randomized, placebo-controlled, cross-over trial of DAR-0100A in patients with SPD using a more comprehensive neuropsychological battery of working memory and verbal learning (as well as those assessing more basic cognitive processes) and serial assessment of serum drug levels. Even within the current limitations, the findings of this study should provide an impetus for the discovery and development of novel $\mathrm{D}_{1}$ agonists that would have improved pharmacokinetic properties, including longer half-life and greater oral bioavailability, which may ultimately be helpful in psychiatric and neurologic disorders characterized by workingmemory impairment, including schizophrenia-spectrum disorders and attention deficit-related disorders.

\section{FUNDING AND DISCLOSURE}

Dr Mailman has a conflict of interest as it relates to existing and pending patents in the $\mathrm{D}_{1}$ agonist area, and has an equity interest in Effipharma that is the assignee of one patent application. He has received compensation in the last 3 years as a consultant in related areas from Pfizer and Roche. His conflict of interests are managed by a standing committee at the Penn State College of Medicine, and he was not involved in the subject testing for this study or the primary data analysis. The following authors have relationships with pharmaceutical companies; specifically: Dr Barch has consulted for Amgen, Roche, and Pfizer and received compensation. Dr Harvey has served as a consultant to AbbVie, Boehringer Ingelheim, En Vivo, Genentech, OtsukaAmerica, Roche, Sunovion, and Takeda Pharmaceuticals.
Dr Harvey is also doing contract research for Genentech. Dr Girgis receives research support from Otsuka Pharmaceuticals. Dr Abi-Dargham consults for UCB, Amgen, and Roche, and receives research support and funding from Forest Laboratories, Takeda Pharmaceuticals, and Pierre Fabre Laboratories. Dr Lieberman serves on the Advisory Board of Intracellular Therapies. He does not receive direct financial compensation or salary support for participation in research, consulting, or advisory board activities. He receives grant support from Allon, Biomarin, Eli Lilly, F. Hoffman-La Roche, Genentech, GlaxoSmithKline, Merck, Novartis, Pfizer, Psychogenics, Sepracor (Sunovion) and Targacept, and he holds a patent from Repligen. The remaining authors declare no conflict of interest.

\section{ACKNOWLEDGEMENTS}

We thank Drs Luis Ripoll and Amir Garakani for medical assistance; Dr Katherine Burdick for statistical consultation; and Nabila Hoque for technical assistance. This work was supported by the Veterans Affairs VISN3 Mental Illness Research, Education, and Clinical Center. Writing of this manuscript was supported by The Department of Veterans Affairs Office of Academic Affiliations Psychiatric Research/ Neurosciences Advanced Fellowship. This publication was made possible by Grant Number MO1-RR-00071 from the National Center for Research Resources (NCRR), a component of the National Institutes of Health (NIH).

\section{DISCLAIMER}

Its contents are solely the responsibility of the authors and do not necessarily represent the official views of NCRR or NIH.

\section{REFERENCES}

Abi-Dargham A (2003). Probing cortical dopamine function in schizophrenia: what can D1 receptors tell us? World Psychiatry 2: 166-171.

Abi-Dargham A, Mawlawi O, Lombardo I, Gil R, Martinez D, Huang Y et al (2002). Prefrontal dopamine D1 receptors and working memory in schizophrenia. J Neurosci 22: 3708-3719.

Arnsten AF, Cai JX, Murphy BL, Goldman-Rakic PS (1994). Dopamine D1 receptor mechanisms in the cognitive performance of young adult and aged monkeys. Psychopharmacology (Berl) 116: 143-151.

Blanchet PJ, Fang J, Gillespie M, Sabounjian L, Locke KW, Gammans $\mathrm{R}$ et al (1998). Effects of the full dopamine D1 receptor agonist dihydrexidine in Parkinson's disease. Clin Neuropharmacol 21: 339-343.

Brewster WK, Nichols DE, Riggs RM, Mottola DM, Lovenberg TW, Lewis $\mathrm{MH}$ et al (1990). Trans-10,11-dihydroxy-5,6,6a,7,8,12bhexahydrobenzo[a]phenanthridine: a highly potent selective dopamine D1 full agonist. J Med Chem 33: 1756-1764.

Buchanan RW, Keefe RS, Lieberman JA, Barch DM, Csernansky JG, Goff DC et al (2011). A randomized clinical trial of MK-0777 for the treatment of cognitive impairments in people with schizophrenia. Biol Psychiatry 69: 442-449.

Darney KJ Jr, Lewis MH, Brewster WK, Nichols DE, Mailman RB (1991). Behavioral effects in the rat of dihydrexidine, a highpotency, full-efficacy D1 dopamine receptor agonist. Neuropsychopharmacology 5: 187-195. 
Egan F, Zhao X, Gottwald R, Harper-Mozley L, Zhang Y, Snavely D et al (2013). Randomized crossover study of the histamine H3 inverse agonist MK-0249 for the treatment of cognitive impairment in patients with schizophrenia. Schizophr Res 146: 224-230.

Garau L, Govoni S, Stefanini E, Trabucchi M, Spano PF (1978). Dopamine receptors: pharmacological and anatomical evidences indicate that two distinct dopamine receptor populations are present in rat striatum. Life Sci 23: 1745-1750.

Gay EA, Urban JD, Nichols DE, Oxford GS, Mailman RB (2004). Functional selectivity of D2 receptor ligands in a Chinese hamster ovary hD2L cell line: evidence for induction of ligandspecific receptor states. Mol Pharmacol 66: 97-105.

George MS, Molnar CE, Grenesko EL, Anderson B, Mu Q, Johnson K et al (2007). A single $20 \mathrm{mg}$ dose of dihydrexidine (DAR-0100), a full dopamine D1 agonist, is safe and tolerated in patients with schizophrenia. Schizophr Res 93: 42-50.

Giardina WJ, Williams M (2001). Adrogolide HCl (ABT-431; DAS431), a prodrug of the dopamine D1 receptor agonist, A-86929: preclinical pharmacology and clinical data. CNS Drug Rev 7: 305-316.

Gilmore JH, Watts VJ, Lawler CP, Noll EP, Nichols DE, Mailman RB (1995). 'Full' dopamine D1 agonists in human caudate: biochemical properties and therapeutic implications. Neuropharmacology 34: 481-488.

Goldman-Rakic PS (1994). Working memory dysfunction in schizophrenia. J Neuropsychiatry Clin Neurosci 6: 348-357.

Goldman-Rakic PS, Castner SA, Svensson TH, Siever LJ, Williams GV (2004). Targeting the dopamine D1 receptor in schizophrenia: insights for cognitive dysfunction. Psychopharmacology (Berl) 174: 3-16.

Graham DL, Hoppenot R, Hendryx A, Self DW (2007). Differential ability of D1 and D2 dopamine receptor agonists to induce and modulate expression and reinstatement of cocaine place preference in rats. Psychopharmacology (Berl) 191: 719-730.

Green MF (2006). Cognitive impairment and functional outcome in schizophrenia and bipolar disorder. J Clin Psychiatry 67: e12.

Harvey PD (2009). Pharmacological cognitive enhancement in schizophrenia. Neuropsychol Rev 19: 324-335.

Isacson R, Kull B, Wahlestedt C, Salmi P (2004). A 68930 and dihydrexidine inhibit locomotor activity and d-amphetamineinduced hyperactivity in rats: a role of inhibitory dopamine $\mathrm{D}(1 / 5)$ receptors in the prefrontal cortex? Neuroscience 124: 33-42.

Kebabian JW, Calne DB (1979). Multiple receptors for dopamine. Nature 277: 93-96.

Keefe RS (2007). Cognitive deficits in patients with schizophrenia: effects and treatment. J Clin Psychiatry 68(Suppl 14): 8-13.

Kilts JD, Connery HS, Arrington EG, Lewis MM, Lawler CP, Oxford GS et al (2002). Functional selectivity of dopamine receptor agonists. II. Actions of dihydrexidine in D2L receptor-transfected MN9D cells and pituitary lactotrophs. J Pharmacol Exp Ther 301: 1179-1189.

Kirrane RM, Mitropoulou V, Nunn M, New AS, Harvey PD, Schopick F et al (2000). Effects of amphetamine on visuospatial working memory performance in schizophrenia spectrum personality disorder. Neuropsychopharmacology 22: 14-18.

Knoerzer TA, Nichols DE, Brewster WK, Watts VJ, Mottola D, Mailman RB (1994). Dopaminergic benzo[a]phenanthridines: resolution and pharmacological evaluation of the enantiomers of dihydrexidine, the full efficacy D1 dopamine receptor agonist. J Med Chem 37: 2453-2460.

Lei G, Anastasio NC, Fu Y, Neugebauer V, Johnson KM (2009). Activation of dopamine D1 receptors blocks phencyclidineinduced neurotoxicity by enhancing N-methyl-D-aspartate receptor-mediated synaptic strength. J Neurochem 109: 1017-1030.

Lovenberg TW, Brewster WK, Mottola DM, Lee RC, Riggs RM, Nichols DE et al (1989). Dihydrexidine, a novel selective high potency full dopamine D-1 receptor agonist. Eur J Pharmacol 166: 111-113.

Mailman R, Huang X, Nichols DE (2001). Parkinson's disease and D1 dopamine receptors. Curr Opin Investig Drugs 2: 1582-1591.

Mailman RB (2007). GPCR functional selectivity has therapeutic impact. Trends Pharmacol Sci 28: 390-396.

McClure MM, Barch DM, Flory JD, Harvey PD, Siever LJ (2008). Context processing in schizotypal personality disorder: evidence of specificity of impairment to the schizophrenia spectrum. J Abnorm Psychol 117: 342-354.

McClure MM, Harvey PD, Goodman M, Triebwasser J, New A, Koenigsberg HW et al (2010). Pergolide treatment of cognitive deficits associated with schizotypal personality disorder: continued evidence of the importance of the dopamine system in the schizophrenia spectrum. Neuropsychopharmacology 35: 1356-1362.

Michaelides MR, Hong Y, DiDomenico S Jr, Asin KE, Britton DR, Lin CW et al (1995). (5aR,11bS)-4,5,5a,6,7,11b-hexahydro-2propyl-3-thia-5-azacyclopent-1- ena[c]-phenanthrene-9,10-diol (A-86929): a potent and selective dopamine D1 agonist that maintains behavioral efficacy following repeated administration and characterization of its diacetyl prodrug (ABT-431). J Med Chem 38: 3445-3447.

Mitropoulou V, Harvey PD, Maldari LA, Moriarty PJ, New AS, Silverman JM et al (2002). Neuropsychological performance in schizotypal personality disorder: evidence regarding diagnostic specificity. Biol Psychiatry 52: 1175-1182.

Mitropoulou V, Harvey PD, Zegarelli G, New AS, Silverman JM, Siever LJ (2005). Neuropsychological performance in schizotypal personality disorder: importance of working memory. Am J Psychiatry 162: 1896-1903.

Mottola DM, Brewster WK, Cook LL, Nichols DE, Mailman RB (1992). Dihydrexidine, a novel full efficacy D1 dopamine receptor agonist. J Pharmacol Exp Ther 262: 383-393.

Mottola DM, Kilts JD, Lewis MM, Connery HS, Walker QD, Jones SR et al (2002). Functional selectivity of dopamine receptor agonists. I. Selective activation of postsynaptic dopamine D2 receptors linked to adenylate cyclase. J Pharmacol Exp Ther 301: $1166-1178$

Mottola DM, Laiter S, Watts VJ, Tropsha A, Wyrick SD, Nichols $\mathrm{DE}$ et al (1996). Conformational analysis of D1 dopamine receptor agonists: pharmacophore assessment and receptor mapping. J Med Chem 39: 285-296.

$\mathrm{Mu}$ Q, Johnson K, Morgan PS, Grenesko EL, Molnar CE, Anderson B et al (2007). A single $20 \mathrm{mg}$ dose of the full D1 dopamine agonist dihydrexidine (DAR-0100) increases prefrontal perfusion in schizophrenia. Schizophr Res 94: 332-341.

Salmi P, Isacson R, Kull B (2004). Dihydrexidine-the first full dopamine D1 receptor agonist. CNS Drug Rev 10: 230-242.

Schneider JS, Sun ZQ, Roeltgen DP (1994). Effects of dihydrexidine, a full dopamine D-1 receptor agonist, on delayed response performance in chronic low dose MPTP-treated monkeys. Brain Res 663: 140-144.

Setler PE, Sarau HM, Zirkle CL, Saunders HL (1978). The central effects of a novel dopamine agonist. Eur J Pharmacol 50: 419-430.

Siegel BV Jr, Trestman RL, O’Flaithbheartaigh S, Mitropoulou V, Amin F, Kirrane R et al (1996). D-amphetamine challenge effects on Wisconsin Card Sort Test. Performance in schizotypal personality disorder. Schizophr Res 20: 29-32.

Slifstein M, Suckow RF, Javitch JA, Cooper T, Lieberman J, Abi-Dargham A (2011). Characterization of in vivo pharmacokinetic properties of the dopamine D1 receptor agonist DAR-0100A in nonhuman primates using PET with [11C] NNC112 and [11C] raclopride. J Cereb Blood Flow Metab 31: 293-304.

Steele TD, Hodges DB Jr, Levesque TR, Locke KW (1997). D1 agonist dihydrexidine releases acetylcholine and improves 
cognitive performance in rats. Pharmacol Biochem Behav 58: 477-483.

Thompson JL, Rosell DR, Slifstein M, Girgis RR, Xu X, Ehrlich Y et al (2014). Prefrontal dopamine D1 receptors and working memory in schizotypal personality disorder: a PET study with [C]NNC112. Psychopharmacology (Berl) doi:10.1007/s00213-0143566-6.

Velligan D, Brenner R, Sicuro F, Walling D, Riesenberg R, Sfera A et al (2012). Assessment of the effects of AZD3480 on cognitive function in patients with schizophrenia. Schizophr Res 134: 59-64.
Watts VJ, Lawler CP, Gilmore JH, Southerland SB, Nichols DE, Mailman RB (1993). Dopamine D1 receptors: efficacy of full (dihydrexidine) $v s$ partial (SKF38393) agonists in primates $v s$ rodents. Eur J Pharmacol 242: 165-172.

Watts VJ, Lawler CP, Gonzales AJ, Zhou QY, Civelli O, Nichols DE et al (1995). Spare receptors and intrinsic activity: studies with D1 dopamine receptor agonists. Synapse 21: 177-187.

Zhang J, Xiong B, Zhen X, Zhang A (2009). Dopamine D1 receptor ligands: where are we now and where are we going. Med Res Rev 29: $272-294$. 